\title{
Contabilidad de costos en la pequeña y mediana industria venezolana del calzado
}

\author{
Maria Anna Amerio* \\ Orlando Viloria" \\ Luis Nava
}

\section{Resumen}

El presente trabajo discute inicialmente la evolución de la contabilidad de costos y contrasta los sistemas de costos tradicionales y los modernos, y su potencialidad para las $P$ y MI. El objetivo fundamental es evaluar las posibilidades de la contabilidad de costos y su aporte a la gerencia en la $P$ y $M I$ venezolana del calzado. La metodología utilizada fue de tipo exploratoria y abarcó: una revisión documental, entrevistas a gerentes del sector y a directivos de la Cámara Venezolana del Calzado, así como la técnica de investigación-acción. En el rol de asesor contable de dos empresas de calzado, se establecieron las siguientes conclusiones: la pequeña y mediana industria del calzado calcula los costos mediante métodos extracontables, los enfoques utilizados en el cálculo de los costos limitan la toma de decisiones, para llegar a la situación ideal se requiere una serie de etapas evolutivas en los sistemas de gestión y contabilidad de coslos, los sistemas mencionados deben desarrollarse en función de la cultura contable existente en la empresa.

Palabra claves: Contaduría, Contabilidad, Costos, Gerencia, Finanzas, Industria del Calzado.

Recibido: 12-06-97 . Aceptado: 27-06-97

Los autores son profesores de la Universidad Simón Bolívar, Sede Litoral.

* Lic. Contaduría Pública, Especialista en Economia Industrial.

** Lic. en Computación, Msc. en Ingenieria de Sistemas.

*** Economista, Msc. en Relaciones Económicas Internacionales

Dirección: Valle de Camurí Grande, Depto. Vargas./ TIf: $031-722911$ al 16 ext 129; 131. FAX: $031-722315$. 


\title{
Cost accounting in the small and medium sized shoe industry in Venezuela
}

\begin{abstract}
This paper begins with a discussion of the evolution of cost accounting and contrasts the traditional and modern cost accounting systems and their potential for use in small (S) and Medium (M) sized industries. The fundamental objective was to evaluate the possibility of cost accounting and its advantages in the management of the $S$ and $M$ shoe industries in Venezuela. The methodology used was exploratory and included: documentary revision, interviews with management personnel and directors of the Venezuelan Shoe Industry Council, and the tecnique of action-research. In the role of accounting advisor to two venezuelan shoe industries, the following conclusions were reached: the $S$ and $M$ venezuelan shoe industries calculate costs by means of non-accounting methods; these methods of calculation of costs limits decision making; in order to achieve an ideal situation, a series of evolutionary steps are needed in management and cost accounting systems; such systems must be developed in relation to the existing accounting practices of each company.
\end{abstract}

Key Words: Accounting, Costs, Management, Finance, Shoe Industry.

\section{Introducción}

Las pequeñas y medianas industrias (PyMI) son un factor para que un país sea competitivo. Al respecto Enright (1994) concluye que las pequeñas y medianas empresas juegan un papel significativo en las economías desarrolladas, ya que generan una porción sustancial de la producción y el empleo. A la par, deben estimular el desarrollo e innovación en todos sus procesos, incluyendo el contable y gerencial, ya que sin la información contable y en particular la de costos, la gerencia no puede tomar decisiones claves en las diferentes áreas de la empresa. En consecuencia, es importante la existencia de sistemas de contabilidad de costos eficientes y efectivos que suministren informes gerenciales de calidad. Es por ello, que es necesario realizar investigaciones en esta área que aporten conocimiento a los gerentes del sector, con la finalidad de hacer de sus empresas organizaciones más competitivas.

Por consiguiente, el presente trabajo forma parte de una investigación relacionada con la implantación de sistemas de contabilidad gerencial en la pequeña y mediana industria en Venezuela. Su propósito es determinar y evaluar la situación actual de los sistemas de contabilidad de costos en la P y MI del sector calzado, debido a las grandes oportunidades que el mismo tiene de insertarse en los mercados internacionales. Para ello se presenta la evolución de los sistemas de contabilidad de costos; las teorias contables existentes en la actualidad y una comparación entre éstas, de acuerdo con 
los expertos del área, con el fin de resaltar las características ideales de los sistemas mencionados.

\section{Evolución de la Contabilidad de Costos}

A medida que se desarrollaron las civilizaciones fueron aumentando las necesidades de bienes y servicios, paralelamente la actividad económica se intensificó y diversificó, pasando, por etapas, de una economía doméstica a una economia de intercambio.

Según explica Lorino (1994) al principio, el propietario de la empresa a menudo gestionaba él mismo su patrimonio y por lo tanto dominaba las técnicas de gestión.

Al surgir las industrias artesanales, en el siglo XI, comenzaba el intercambio comercial de los productos; los dueños requerían información rudimentaria sobre los precios, los costos relacionados e ingresos percibidos, con el fin de determinar sus ganancias; lo cual en esa época era simple de calcular, según analiza Polimeni (1994).

A medida que las empresas se desarrollaron a partir del siglo $X I X$ con la revolución industrial, su estructura organizacional evolucionó. Las empresas familiares de los siglos XVIII y XIX fueron reemplazadas paulatinamente por pequeñas, medianas y grandes empresas más organizadas; $y$ algunas con estructuras organizacionales complejas. En consecuencia, se hizo necesario determinar el costo de los productos en cada fase del proceso de producción y medir el desempeño de los trabajadores y gerentes en todas las etapas, esto obligó a su vez a realizar cálculos complicados para determinar el costo total.

Posteriormente surge la contabilidad analítica, propuesta por Taylor, Emerson y Harrison entre otros, que incluye, según señala Lorino (1994:8): "la asignación de los costos indirectos, el cronometraje y el seguimiento de los tiempos de mano de obra directa, los estándares, la asignación de los costos indirectos mediante los tiempos de mano de obra o de los tiempos de máquina, la gestión de las existencias y de las materias primas y la remuneración del rendimiento" . Este sistema se adaptó satisfactoriamente al entorno económico existente en esa época, siendo la razón fundamental el estado estático de las industrias.

Evidentemente, hoy en día la actividad empresarial es cada vez más dinámica si consideramos los planteamientos de Catacora (1996), el cual señala que lo único permanente en la actualidad es el cambio, debido al desarrollo intensivo de las tecnologías, al menor ciclo de vida de los productos y a la fuerte competencia internacional. Por consiguiente los sistemas de costos diseñados para una época, hoy quizás no produzcan los mismos resultados.

En tal sentido, Polimeri (1994:3) afirma: "La importancia de adaptar la contabilidad de costos para satisfacer las necesidades de nuevos ambientes comerciales, es clara a partir del actual desafío que enfrentan los contadores de costos".

Por otro lado, Lorino (1994:4) en concordancia con Alvarez (1994) señala que la industria y los procesos industriales cam- 
bian, por lo tanto los controles de gestión deben cambiar con la misma velocidad. Por esta razón, menciona: "Conocer y aplicar lo más actual en este ámbito es necesario y urgente. Necesario, porque las técnicas de control se han convertido en una ventaja competitiva determinante y en un campo de innovación decisivo."

En consecuencia las teorias relacionadas con la contabilidad de costos se desarrollaron a través del tiempo, aunque no con la misma velocidad y variedad que la tecnología y los sistemas gerenciales, ésto se refleja al revisar los conceptos presentados por expertos durante los últimos veinte años.

Neuner (1979:3) expone un concepto tradicional: "La contabilidad de costos es, por consiguiente, una fase amplificada de la contabilidad general o financiera de una entidad industrial o mercantil, que proporciona rápidamente a la gerencia los datos relativos a los costos de producir o vender cada artículo o de suministrar un servicio en particular".

A su vez, destaca que lo importante de esta rama de la contabilidad es suministrar el costo de cada producto que se vende o servicio que se presta; por lo tanto los datos suministrados a la gerencia se relacionan con la mano de obra, carga fabril, gastos de administración y ventas de cada producto o servicio.

Con el tiempo surgió la contabilidad gerencial. En tal sentido la National Association of Accountants (NAA) en el Statement on Management Accounting (SMA) No. 1A, la define como el proceso que comprende la identificación de las transacciones comerciales $u$ otros hechos económicos; la medición, la cual incluye estima- ciones de las transacciones comerciales $u$ otros hechos económicos que se han causado o puedan causarse; la acumulación de manera ordenada y coherente para el registro y clasificación de los mismos; el análisis, preparación e interpretación de datos presentados en forma lógica con el fin de satisfacer una necesidad de información $y$ comunicación a la gerencia y otras personas, para uso interno y externo.

Por su parte Horngren (1996) expone que los sistemas de contabilidad gerencial comprenden los sistemas de acumulación de costos, los presupuestos, análisis de variaciones e información del desempeño.

Analizando los conceptos expuestos, se deduce que actualmente la contabilidad de costos es una herramienta para determinar los costos de un proyecto, proceso, actividad, producto o servicio; y la contabilidad gerencial es un proceso que utiliza la contabilidad de cosios, además incluye el análisis, preparación e interpretación de datos y la elaboración de informes cuya finalidad es suministrar a la gerencia información relevante para el proceso de toma de decisiones.

Para ello, los expertos afirman que los sistemas de contabilidad gerencial deben suministrar información a valor histórico, actualizado y proyectado, y clasificarla en varias categorías con el fin de satisfacer los requerimientos de los diferentes niveles gerenciales. Los valores históricos y actualizados permiten evaluar el desempeño y los valores proyectados son indispensables en el proceso de toma de decisiones gerenciales tanto a corto como a largo plazo.

Adicionalmente, Alvarez (1994) explica que la contabilidad gerencial se re- 
laciona con la contabilidad financiera suministrando información útil para el proceso de toma de decisiones a corto plazo. A su vez alimentan a la contabilidad de dirección estratégica conformando un triángulo contable, donde el contador debe desarrollar información relevante y útil a la gerencia en el proceso de toma de decisiones a largo plazo.

En el cuadro No. 1, se presenta la situación ideal de un sistema de contabilidad para las empresas en base a Alvarez (1994).

\section{EI Sistema de costos tradicional vs El sistema de costos moderno}

Las teorias analizadas ofrecen dos tipos de sistemas generales. El sistema de asignación de costos tradicional basado en el "volumen" de producción y el sistema basado en "actividades".

Desde el punto de vista tradicional los elementos básicos a considerar para determinar el costo de los productos, de acuerdo con los expertos, son la materia prima, mano de obra directa y costos indirectos de fabricación. Los costos indirectos de fabricación totales de un período, son asignados al costo unitario de los productos utilizando un factor constante de aplicación: horas de mano de obra directa, costo de la mano de obra directa y costo de la materia prima.

El enfoque moderno de acumulación de costos se centra en el sistema de costos basados en actividades (ABC), propuesto entre otros autores, por Kaplan (1994) y Lorino (1993). En este sistema,

Cuadro No. 1

Situación ideal de un sistema de contabilidad

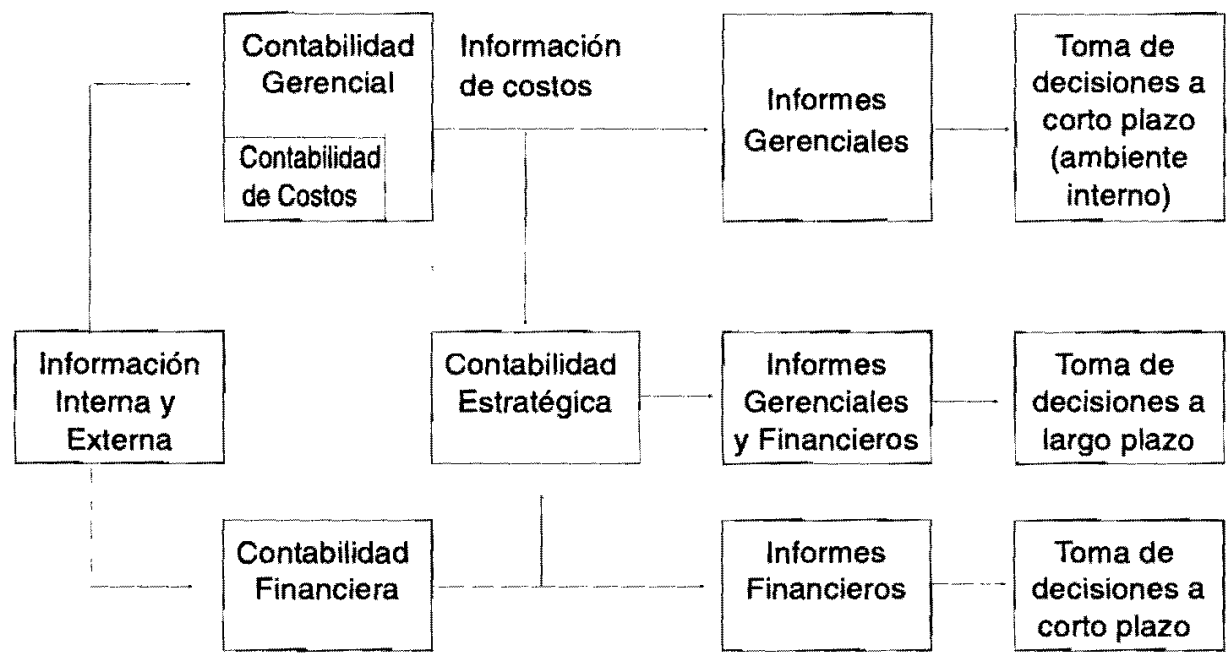

Información

Financiera 
se consideran tanto los procesos como los productos, se analizan los procesos para determinar qué tipos de actividades se desempeñan en relación con el diseño, ingeniería, manufactura, ventas y servicio para cada producto. Las actividades son las que absorben los costos y son definidas y asociadas con productos utilizando el concepto de generadores de costos.

Las actividades se combinan en cadenas o redes de actividades denominadas procesos, destinadas al logro de un objetivo global, es decir, a una salida global material o inmaterial. Al respecto, Lorino (1993) describe el proceso, como el tratado de unión entre los objetivos de la empresa y el desarrollo concreto de las actividades.

Por otra parte la información suministrada por los sistemas de costos basado en actividades es útil y oportuna en empresas donde la gerencia se orienta al mejoramiento continuo, justo a tiempo y la calidad total.

Gran cantidad de expertos como Kaplan (1994), Lorino (1993), Andersen (1996) y Horngren (1996) presentan sus estudios sobre las desventajas de los sistemas de contabilidad de costos tradicional y los efectos negativos en las empresas debido a que no proveen información útil y confiable en la actualidad. Proponen y fomentan la implementación de los sistemas $(A B C)$, alegando que debido al avance tecnológico, la estructura global de los costos industriales cambió durante los últimos veinte años. La proporción de la mano de obra directa disminuyó de más del $\mathbf{5 0} \%$ del valor añadido a menos del $25 \%$, la proporción de los costos indirectos aumentó a un $75 \%$ y los cálculos tradicionales no distribuyen los gastos indirectos de manera equitativa.
A través del sistema mencionado, los costos indirectos se analizan exactamente y se identifican sus verdaderos factores de variabilidad; la mayor parte de ellos pueden asignarse basándose en su consumo efectivo y en consecuencia la asignación a los productos se hace real, se logra un análisis más preciso de las utilidades por producto, cliente, proceso y departamento, y la evaluación del desempeño mejora notablemente. Además, el sistema informa sobre las actividades fundamentales de toda la empresa y los generadores de costos, permitiendo enfocar la atención hacia las causas de los costos, lográndose detectar y eliminar, mediante su análisis, aquellas actividades que no agregan valor. De esa manera se logran mayores niveles de calidad $y$, en consecuencia productividad con el fin de alcanzar y sostener ventajas competitivas.

Es importante considerar que durante el diseño e implantación del sistema $(A B C)$ se pueden presentar dificultades en la definición de las actividades, en la selección de los factores de costos y en la adaptación por parte del personal involucrado

\section{Los Sistemas de Contabilidad de Costos en la Pequeña y Mediana Industria Venezolana}

El sector empresarial venezolano está conformado por un alto porcentaje de PyMI, representando el principal generador de empleo e impulsor de la economía del país. sin embargo, la globalización de los mercados obliga a competir internacionalmente y sobrevivir en un ambiente dinámico donde lo más importante es la innovación. Para ello es necesario que los gerentes de las empresas tomen decisiones 
rápidas y oportunas, apoyados por información relevante financiera y de costos.

Empresas de otros países, como por ejemplo Estados Unidos y Japón, se encuentran altamente desarrolladas en el área gerencial y contable, existiendo interrelación entre la contabilidad gerencial y financiera y el proceso de toma de decisiones, en los tres niveles gerenciales. Según encuestas realizadas por Horngren (1996), un alto porcentaje de ellas sustituyeron el sistema de contabilidad de costos que poseían, por el (ABC). Sin embargo, otras to utilizan fuera de la contabilidad, en forma paralela a la contabilidad de costos existente en las mismas, como complemento para el análisis de las actividades.

Pareciera lógico que las empresas venezolanas que aspiren competir internacionalmente sigan las mismas tendencias. En la práctica esto no es tan sencillo, debido a la cultura contable existente en la PyMl del país.

La condición de los sistemas contables en las empresas venezolanas, en base a investigaciones realizadas por $\mathrm{Ka}$ plan (1994), Enright (1994) y Catacora (1996), no proporcionan registros internos formales relacionados con contabilidad de costos. Kaplan particularmente explica que esta situación se debe a las condiciones que existían en el país antes de la apertura comercial, donde las empresas contaban con un mercado cautivo protegido por barreras arancelarias, permitiéndoles trasiadar el aumento de los costos por ineficiencia a los productos finales, no afectándose su capacidad competitiva. En tal sentido, Amerio (1996) señala que un grupo significativo de $P$ y MI no poseen internamente sistemas de contabilidad financiera y de costos que suministren información útil en el proceso de toma de decisiones gerenciales.

Analizando la evolución de la contabilidad de costos y comparando la situación de la PyMI venezolana se concluye que en el país estas industrias no se han desarrollado contablemente, y existe una gran brecha entre su cultura contable y las teorias existentes en la actualidad.

\section{La pequeña y Mediana Industria del Sector Calzado}

La producción del calzado en Venezuela constituye una de las actividades más importantes del grupo de la PyMI.

Al respecto Antolinez(1995), destaca la posibilidad de competir positivamente en los mercados internacionales, resaltando como fortalezas la existencia de un conglomerado cuantitativamente significativo de PyMI , 10 que asigna mayor flexibilidad para adaptarse a los cambios en el entorno.

Además de la existencia de gerentes expertos en la actividad productiva y comercial, trabajadores capacitados, diseños y manufacturas de buena calidad y tecnologías relativamente actualizadas. $S$ in embargo, puede verse afectada por la cultura contable existente, los sistemas de costos utilizados y las técnicas de gestión.

En tal sentido, se realizó la investigación de la situación actual de los sistemas de costos, utilizando una metodología de tipo exploratoria, ya que se consultaron diversas fuentes bibliográficas $y$ estadísticas de la OCEl; se aplicaron entrevistas a los miembros de la Cámara Venezolana del Calzado, a gerentes de empresas del sector y se utilizó la técnica 
de investigación acción en el rol de asesor contable en dos de ellas.

El estudio realizado arrojó los siguientes resultados:

\section{1.- Aspectos Generales}

- Inexistencia de sistemas formales de contabilidad para el procesamiento de los datos de costos.

- Inexistencia de presupuestos formales en el área de costos.

- Adquisición de sistemas administrativos automatizados sin lograr su implantación.

2.- Estructura de costos del sector

- Mano de obra:46,5\%, Materia Prima:48,4\% y gastos indirectos de fabricación: $5,3 \%$.

3.- Determinación de los costos

- El cálculo de los costos no se realiza a través de métodos contables, sino mediante métodos matemáticos simples.

- El objetivo principal es determinar el costo unitario por producto terminado o línea de producto terminado en base a un período dado.

- Los costos que se calculan son estimados.

- Los datos utilizados son: materia prima requerida por producto, mano de obra por hora promedio trabajada, total de gastos indirectos de fabricación promedio por periodo en base a un porcentaje, índice de inflación.

4.- Valoración de Inventarios

- La valoración de los inventarios se realiza de manera periódica (anual), a través de inventarios físicos.

\section{5.- Informes a la gerencia}

- Se concentran en una ficha detallada, la cantidad y costo estimado de materia prima, mano de obra, asi como los gastos para un producto o línea de producto.

6.- Objetivo de la información para la gerencia.

- Conocer el costo estimado de elaboración de los productos y establecer de esa manera el precio de venta, considerando los de la competencia.

- Determinar la utilidad bruta estimada por producto en base a precios de venta reales y costos estimados.

- Cumplir con las exigencias de organismos gubernamentales como la OCEI, Fomento y Ministerio de Hacienda.

Analizando las condiciones de los sistemas de costos existentes en el sector, se deduce la tendencia a determinar los costos bajo el enfoque tradicional según las teorías de Taylor, pero sin utilizar un modelo de contabilidad formal y sistemas automatizados que suministren informes adecuados, lo que trae como consecuencia la inexistencia de contabilidad gerencial como apoyo en el proceso de toma de decisiones.

Por lo tanto, los gerentes en el sector tienden a tomar decisiones de manera intuitiva, lo cual encaja perfectamente con las conclusiones de Burgos (1994), en cuanto a los métodos utilizados por los gerentes venezolanos. 
Por otra parte, no puede medirse el desempeño de la empresa y realizarse un control sobre las operaciones, debido a que no existe información sobre los costos reales incurridos en el período.

\section{Conclusiones y Recomendaciones}

El grupo representativo del sector estudiado no posee un sistema formal para procesar los datos de costos. Llegar a la situación ideal requiere una serie de etapas evolutivas en los sistemas de contabilidad y de gestión, con el fin de lograr que la información contable sea una herramienta indispensable para gerenciar en un entorno competitivo.

Los sistemas de contabilidad gerencial deben desarrollarse en función de la cultura contable existente en la empresa y de esa manera lograr integrarse a la organización. Un camino para lograr este desarrollo puede ser el mejoramiento continuo.

Bajo este esquema, en primer lugar se debe seleccionar a las personas claves de la organización, explicarles el objetivo que se persigue y conducirlas a creer en que "el mejoramiento de todo trabajo y de todos los procesos es a través de calidad para siempre y actuando primero sobre el sistema. Para ello es necesario el deseo de hacerlo, creer que puede hacerse, obtener los medios para hacerio y finalmente iHacerlol" (Conway 1990) citado por Burgos (1995:25).

Una vez alcanzada esta primera fase y lograda la aprobación sincera se cubren los siguientes pasos:

- Creación de un equipo de trabajo con un asesor contable y las personas claves de las diferen- tes áreas de la empresa, considerando que los costos se producen en toda la organización.

- Elaboración de un plan realista, por parte del equipo, tomando como tema principal: "Desarrollo del modelo de contabilidad de costos". El plan debe contener las etapas y los períodos de evolución con los objetivos que se deseen alcanzar a través del mejoramiento continuo, basándose en la metodología presentada por Burgos. Deben incluirse, el tiempo y los costos involucrados en el proceso.

- En la primera etapa se describe y analiza la situación actual de los sistemas de costos expuesta; considerando los materiales, equipos, personal, ejecución y tiempo involucrados en el proceso.

- Posteriormente se elabora y propone un modelo de contabilidad de costos formal, pero sencillo, adaptado a las condiciones internas actuales de la empresa y orientado hacia un enfoque determinado. En tal sentido, la estructura de costos del sector permite el uso de la contabilidad de costos basada en el volumen, ya que según las teorias, no distorsiona los costos por producto. De todas maneras hay que tomar en cuenta la importancia a nivel gerencial de analizar los costos de las actividades. La decisión depende de los gerentes.

- Paralelamente al modelo propuesto, es necesario crear, con 
la participación del personal, un manual de normas y procedimientos que comprenda la descripción, el flujograma del proceso, del flujo de datos a través de las diferentes áreas, los formularios a utilizar, su registro, la elaboración de informes y el uso por parte de la gerencia. El gerente por su parte debe familiarizarse con las técnicas de análisis y sus objetivos.

- La siguiente etapa es introducir el proceso de mejoramiento continuo, el cual consiste en desarrollar nuevos procesos, mejorando y complementando el modelo y orientándolo, si la cultura gerencial lo permite, hacia los enfoques modernos, $y$ convertirlo en un sistema de contabilidad gerencial eficiente. Es importante destacar que en el mejoramiento continuo no se requieren grandes inversiones de dinero, ni cambios bruscos en los procesos.

- En definitiva, en cada etapa deben evaluarse los resultados y determinar la eficiencia del modelo a través de la satisfacción del gerente por la calidad de información gerencial suministrada.

\section{Referencias Bibliográficas}

Alvarez, José; Amat, Joan; Balada Tomás; Blanco, Felipe; Castelló Emma; Lizcano, Jesús (1994). Introducción a la Contabilidad de Gestión. Editorial McGraw-Hill, 291p. España.
Amerio, Maria (1996), Importancia de la contabilidad de costos en la PyMI, Revista Espacios. Vol 2. Conicit. Venezuela.

Andersen, Arthur (1996). El Costeo Basado en Actividades. Revista Reinventando el Costo. Vol 1 (4-9). Venezuela.

Antolinez, Roberto (1995). Calzado en la Mira. Revista Dinero. Venezuela.

Burgos, Ignacio (1994). Decisión ¿ Laberinto Gerencial?. UCV, 165p. Venezuela.

Burgos, Ignacio (1995). Guía Mejoramiento Continuo. Presentado en: Charia Gerencia Productividad USM. Caracas. Venezuela.

Catacora, Fernando (1996). Sistemas y Procedimientos Contables, Editorial McGraw-Hill , p.192-208. Venezuela.

Enright, Michael; Francés, Antonio (1994). Venezuela: el reto de la competitividad, Ediciones IESA, p.617-656. Venezuela.

Horngren, Charies; Foster, George; Srikant, Datar (1996), Contabilidad de Costos un enfoque gerencial, Editorial Prentice Hall p. 140-169. México.

Kaplan, Robert (1994). La Revolución Contable. El sistema de Costos Basado en Actividades: Una herramienta estratégica. Seminario, Centro de Finanzas del IESA. Caracas. Venezuela.

Lorino, Philippe (1993); El control de gestión eatratégico, Ediciones Marcombo S.A., p.193. Barcelona.

Neuner, John (1979) Contabilidad de Costos. Principios y Práctica. Ediciones UTEHA. Cap. 1. México.

Polimeni, Ralph; Fabozzi, Frank; Adeiberg, Art hur (1994). Contabilidad de Costos Conceptos y Apllcaciones para la toma de Decisiones Gerenciales, Editorial Nomos S.A., p. 780 . Colombia. 